\title{
Rehearsal and retrieval processes in free recall of categorized lists
}

\author{
MARK H. ASHCRAFT, GEORGE KELLAS, and SHERRY NEEDHAM \\ University of Kansas and Bureau of Child Research, Lawrence, Kansas 66045
}

\begin{abstract}
The relationships between rehearsal and subsequent retrieval characteristics were examined in the context of free recall of categorized lists. The results indicated a direct correspondence between the frequency of rehearsal and the order and speed of retrieval within categories. The same relationship obtained for the categories themselves. It was suggested that both retrieval time and order effects can be predicted in terms of the organization of input processing and the resultant repetition frequency of categories and exemplars.
\end{abstract}

Kellas, Ashcraft, Johnson, and Needham (1973) have recently proposed an hypothesis concerning storage and retrieval in the free recall of categorized lists. The hypothesis maintains that retrieval order and speed are determined by the organized repetitions of list items and the categories to which they belong. Specifically, patterns of input processing time (Kellas et al., 1973) and overt rehearsal (Rundus, 1971, Experiment IV) suggest that conceptually related items are unitized into chunks, with previously stored instances of a category being retrieved and rehearsed as later instances are presented. This differential rehearsal of category items then affects retrieval within the category such that the most frequently rehearsed item is retrieved earliest and with the shortest interword response time. (IRT).

Retrieval of higher order units is assumed to operate on the same basis; that is, higher order units whose elements received the most overall rehearsal will be retrieved earlier and more rapidly than units which were rehearsed less. Accordingly, the hypothesis predicts fewer repetitions for category $n+1$ than for category $n$ in recall, and fewer repetitions for item $i+1$ than $i$ within any recalled category. This direct relationship between repetition and retrieval order is modified such that recalling all of a category's accessible items takes priority over the absolute rehearsal frequency of individual items. In other words, an additional prediction is that the last recalled item in category $n$ will have received fewer repetitions than the first recalled item in $\mathrm{n}+1$ (see also Rundus, 1973). The predictions for retrieval time follow directly, with increased rehearsal resulting in more rapid retrieval.

A critical difference exists between the present hypothesis and the random sampling with replacement model proposed by Patterson, Meltzer, and Mandler

The present investigation was partially supported by PHS Grants HDO0183 and HDO0870 from the National Institute of Child Health and Human Development to the Bureau of Child Research, and a General Research Grant (No. 3331) fxom the University of Kansas to the second author. A portion of the present data provided the basis of a thesis submitted by the third author to the University of Kansas. Reprint requests should be addressed to George Kellas, Department of Psychology, University of Kansas, Lawrence, Kansas 66045.
(1971). These investigators examined the temporal characteristics of categorized recall, and found an exponential increase in the function describing higher order unit retrieval. To account for this increase, the Patterson et al. model asserts that both items and categories are randomly drawn from their respective pools, are subjected to a recognition check for possible previous output, and are always replaced into the sampling pools after being drawn. Under these circumstances, the probability of sampling a previously recalled unit would increase as output continues. This increase, in turn, results in more rejections of sampled units later in output, and an exponential increase in the retrieval time function. The present hypothesis differs from this model in two ways. First, the recognition component which yields exponentiality is assumed to operate when subjects have not been allowed sufficient time to reorganize the list and develop a systematic retrieval plan. Using a subject-paced input procedure, Kellas et al. (1973) found active reorganization of the list items, with no evidence for exponentiality in the retrieval time functions. Secondly, Patterson et al. (1971) proposed that the order of retrieval within and across categories is randomly determined, whereas our hypothesis maintains that the order of output should be a direct function of rehearsal frequency and organization.

The present investigation was designed to broaden the empirical base of information concerning storage and retrieval processes in the free recall of categorized lists. Accordingly, study times, overt rehearsals, and retrieval times were recorded in order to supplement more typical measures of performance. The a priori degree of list organization was manipulated so that the reorganization of category instances could be examined in relation to rehearsal and recall. It was expected that the patterns of overt rehearsal would be directly related to the order and speed of retrieval for all sequential organization groups, with categories and items within categories being recalled on the basis of repetition frequency. Such a demonstration would be difficult to explain within a random sampling framework. 
It was expected that patterns of overt rehearsal would reflect covert rehearsal processes, and thereby provide a basis for interpretation of the study and retrieval time profiles. However, the extent to which externalized rehearsal interferes with normal rehearsal processes is unclear. Fischler, Rundus, and Atkinson (1970) compared the free recall of unrelated words under overt and covert rehearsal conditions, and found superior performance for covert groups. Given that externalizing silent rehearsal requires additional time, these results might have been predicted for an experimenter-paced task. Kellas, McCauley, and McFarland (1975), using a subject-paced procedure with unrelated words, reported larger time increments across input positions for overt vs. covert conditions, with no differences in the level of correct responding. Neither Fischler et al. (1970) nor Kellas et al. (1975) suggest what effect overt rehearsal would have on lists of semantically related items, although Kellas et al. indicated that the overt rehearsal requirement increased serial processing in the free recall task. Consequently, a covert rehearsal control condition was included to assess more closely any changes in acquisition which might result from the required verbalization. A serial recall requirement was also included to provide a standard against which free recall performance could be evaluated. Comparisons between free and serial recall for both rehearsal conditions provided the requisite data to determine the extent to which overt rehearsal increased the tendency to process categorized lists in a serial fashion.

\section{METHOD}

\section{Subjects}

One hundred and twenty undergraduates, enrolled in introductory psychology courses at the University of Kansas, participated for class credit. Subjects who had previously participated in verbal learning experiments were excluded.

\begin{abstract}
Materials and Lists
Four exemplars from each of 28 categories were selected from the Battig and Montague (1969) norms. For all categories, the exemplars represented the most frequent responses to the category names. Seven lists were constructed by randomly sampling, without replacement, four categories successively until the sample was exhausted. Consequently, there were seven lists of four categories with four items per category. The first list was treated as a practice list and was not included in any analy ses.

For each of the lists, a priori degree of organization was manipulated by varying the number of times a word was followed by another word from the same category. The three levels of sequential organization were 4-block, 2-block, and random. By designating the four categories per list by the letters A through $D$, the following represent examples of the 4- and 2-block lists, respectively: AAAABBBBCCCCDDDD; $A A B B C C D D A A B B C C D D$. Item order was constrained in the random condition such that (a) no two instances of the same category occurred consecutively and (b) one exemplar from each category appeared in each successive block of four items.
\end{abstract}

\footnotetext{
Apparatus

Stimulus items were typed onto transparency paper and mounted in Easymount $35 \mathrm{~mm}$ frames. A Sawyer Rotomatic
}

slide projector was used to present the stimuli on a daylight screen. A response button was programmed to the projector such that the subject controlled the viewing time of each slide. The latency between the button response and the visual presentation of each stimulus was approximately $.8 \mathrm{sec}$. The duration of each presentation was automatically presented to the nearest $.05 \mathrm{sec}$ by means of a pulse stream generator, parallelentry control panel, and a Sodeco printout counter. The subjects' overt rehearsal and oral recall were tape recorded. Interword response times (IRTs) were measured by feeding the taped recall into a Mechanics for Electronics heatwriting graphic recorder (Model 20C-AHA). The subject's recall actuated a pen which indicated word onset and offset on paper moving at the rate of $25 \mathrm{~mm} / \mathrm{sec}$. All IRTs were calculated to include only the time between words, and to exclude word production time.

\section{Design and Procedure}

The subjects were randomly assigned to the cells of a 2 by 2 by 3 factorial matrix representing recall requirement (free and serial), rehearsal requirement (overt and covert), and three levels of sequential organization (4-block, 2-block, and random)..Ten subjects served in each unique combination of the treatment conditions.

The subjects were tested individually. Half of the subjects were given instructions requiring serial recall, and half received standard free recall instructions. For both recall requirements, half of the subjects were told that they must rehearse overtly. There were no restrictions placed on the choice of items to be rehearsed or the rate of rehearsal; subjects were simply asked to rehearse throughout the entire exposure period. The remaining subjects were asked to rehearse covertly. All subjects were given instructions regarding the subject-paced presentation, and were told that a red slide would serve as the signal for recall. The subjects were given unlimited time for recall, but were asked to indicate that they had finished recall before going on to the next list.

\section{RESULTS AND DISCUSSION}

\section{Replicated Effects}

The results of the present investigation essentially replicated the comparable effects reported by Kellas et al. (1973); consequently, only a synopsis of the replicated effects will be presented (all described effects reached at least the .05 level of significance). Different levels of a priori list organization resulted in differential increases in study time. The 4-block groups exhibited a pattern of peaks at category boundary positions, suggesting that subjects paused at the category boundaries to rehearse the items according to category membership. The study time increases in the 2-block and random free recall conditions indicated that subjects in these groups reorganized the list items according to category membership during presentation, with less reorganization time and rehearsal required for the 2-block groups due to the greater degree of list organization. Size of recalled clusters decreased as a priori list organization decreased; average cluster size was $3.68(\mathrm{SD}=0.37), 3.44 \quad(\mathrm{SD}=0.51)$, and 2.89 $(\mathrm{SD}=1.06)$, for 4-block, 2-block, and random groups, respectively. Cluster size also decreased across output positions. It should be noted that the extent of clustering shown here supports the notion of 
reorganization during input; that is, cluster sizes for 2-block and random groups exceeded the values (2.0 and 1.0 , respectively) which would have been expected if these groups had recalled items according to input order. Finally, temporal analysis of retrieval indicated no sequential organization effects on within-category IRTs, but a reliable increase in between-category IRTs as list organization increased.

\section{Rehearsal and Retrieval Relationships}

The results of primary interest were those which illustrated the relationships between rehearsal and retrieval characteristics. A brief description of the recall and rehearsal requirement effects, not manipulated in the previous report, is necessary to substantiate inferences based on overt rehearsal data.

Overt rehearsal led to longer study times $[F(1 / 108)=25.03, p<.001, s \bar{x}=2.17]$ and a greater increase in study time across serial positions $[F(15 / 1620)=12.82, p<.001 . \quad s \bar{x}=.13]$ than did covert rehearsal. Means and standard deviations for this interaction are displayed in Table 1a. While this effect suggests that overt rehearsal increased the tendency to process list items in a serial fashion, there was no indication of a differential effect of rehearsal conditions on other factors or interactions (all $F s<1$ ). Correct responding was higher for overt $(\overline{\mathrm{x}}=14.51)$ than covert $(\bar{x}=13.67)$ rehearsal groups $[F(1 / 108)=6.35, p<.025$, $\bar{s} \bar{x}=.41]$ although recall was above $75 \%$ on all trials for all groups. There were no significant effects of rehearsal requirements on cluster size. It appears, then, that overt rehearsal data do provide a valid estimate of covert processing in the present task, with the qualification that a serial processing tendency was more pronounced in the overt rehearsal groups.

Serial recall yielded results similar to those of overt rehearsal on measures of input processing. Serial recall led to longer study times $[\mathrm{F}(1 / 108)=21.79, \mathrm{p}<.001$, $\overline{\mathrm{X}}=2.17]$ and a greater increase across serial positions $[\mathrm{F}(15 / 1620)=3.71, \mathrm{p}<.001, \mathrm{~s} \overline{\mathrm{x}}=.13]$ than did free recall (see Table 1, Part B). This effect, however, did not differentially alter the patterns of study time for sequential organization groups $(\mathrm{Fs}<1$ ), suggesting that serial processing was augmented by an organizational strategy as the level of organization increased in the lists. The analysis of repetitions showed that serial recall groups repeated list items more than free recall groups $[\mathrm{F}(1 / 54)=9.53, \mathrm{p}<.005, \mathrm{~s} \overline{\mathrm{X}}=2.59]$, particularly early list items $\left[\mathrm{F}(15 / 810)=1.79, \mathrm{p}<.05, \mathrm{~s}_{\mathrm{X}}=.09\right]$. The interaction is summarized in Table 1 , Part $\mathrm{C}$. There were no significant effects of recall requirement on the level of correct responding.

The analysis of rehearsal set size allowed a more direct examination of the distribution of item rehearsal across input positions. Rehearsal set size (Rundus, 1971) reflects the number of different items rehearsed at a given input position, i.e., if the subject says "dog, cat, cow, horse" when viewing "horse", his rehearsal set size equals 4 . Thus, if later presented category members

Table 1

Means and Standard Deviations of Input Times (in Seconds) and Overt Rehearsals for Significant Interactions With Serial Positions (1-16)

\begin{tabular}{|c|c|c|c|c|c|c|c|c|c|c|c|c|c|c|c|c|c|}
\hline \multirow[b]{2}{*}{ Effect } & & \multicolumn{16}{|c|}{ Serial Position } \\
\hline & & 1 & 2 & 3 & 4 & 5 & 6 & 7 & 8 & 9 & 10 & 11 & 12 & 13 & 14 & 15 & 16 \\
\hline & & \multicolumn{16}{|c|}{ A. Input Time } \\
\hline Overt & Mean & 4.4 & 4.9 & 6.0 & 9.0 & 9.1 & 11.7 & 115 & 16.0 & 15.1 & 17.7 & 16.2 & 23.5 & 17.8 & 19.7 & 18.6 & 21.6 \\
\hline Rehearsal & $\mathrm{SD}$ & 2.7 & 2.4 & 3.3 & 4.5 & 5.1 & 6.7 & 6.9 & 8.4 & 10.2 & 12.1 & 11.0 & 23.9 & 11.2 & 12.4 & 11.8 & 12.2 \\
\hline Covert & Mean & 4.2 & 4.1 & 5.2 & 7.2 & 7.4 & 7.7 & 8.5 & 10.6 & 9.9 & 10.3 & 9.3 & 11.6 & 10.3 & 9.1 & 9.6 & 11.2 \\
\hline \multirow[t]{2}{*}{ Rehearsal } & $\mathrm{SD}$ & 1.9 & 2.2 & 3.6 & 5.2 & 5.7 & 5.9 & 7.0 & 9.0 & 8.5 & 9.7 & 8.7 & 10.1 & 9.1 & 9.3 & 8.4 & 9.8 \\
\hline & & \multicolumn{16}{|c|}{ B. Input Time } \\
\hline Free & Mean & 3.6 & 3.5 & 4.6 & 6.2 & 6.2 & 7.5 & 7.6 & 10.2 & 9.9 & 10.3 & 9.4 & 14.8 & 11.1 & 11.3 & 10.8 & 11.9 \\
\hline Recall & SD & 1.8 & 1.6 & 2.9 & 3.8 & 4.3 & 5.8 & 6.4 & 8.4 & 9.4 & 11.0 & 10.1 & 23.8 & 10.7 & 11.1 & 10.8 & 10.2 \\
\hline Serial & Mean & 5.0 & 5.4 & 6.6 & 10.0 & 10.3 & 12.0 & 12.4 & 16.3 & 15.1 & 17.7 & 16.0 & 20.3 & 17.1 & 17.5 & 17.4 & 20.8 \\
\hline \multirow[t]{2}{*}{ Recall } & $\mathrm{SD}$ & 2.5 & 2.6 & 3.6 & 5.2 & 5.8 & 6.7 & 6.9 & 8.9 & 9.3 & 11.0 & 9.8 & 12.9 & 10.2 & 12.4 & 10.6 & 12.5 \\
\hline & & \multicolumn{16}{|c|}{ C. Overt Repetitions } \\
\hline Free & Mean & 18.6 & 17.7 & 16.0 & 14.7 & 13.6 & 12.7 & 11.5 & 10.2 & 9.4 & 7.7 & 7.2 & 5.9 & 5.5 & 4.4 & 3.5 & 2.2 \\
\hline Recall & $\mathrm{SD}$ & 13.6 & 12.6 & 12.1 & 10.7 & 9.3 & 9.5 & 8.8 & 8.0 & & 5.8 & 6.7 & 5.1 & 4.8 & 3.6 & 2.8 & 1.3 \\
\hline Serial & Mean & 26.0 & 25.0 & 22.3 & 19.8 & 19.7 & 17.9 & 16.4 & 15.2 & 16.2 & 14.8 & 14.0 & 11.7 & 11.4 & 9.4 & 7.2 & 4.4 \\
\hline \multirow{2}{*}{ Recall } & $\mathrm{SD}$ & 11.9 & 12.8 & 11.3 & 10.2 & 10.8 & 9.6 & 9.1 & 8.6 & 8.4 & 7.7 & 8.3 & 7.3 & 6.7 & 5.3 & 4.3 & 2.5 \\
\hline & & \multicolumn{16}{|c|}{ D. Rehearsal Set Size } \\
\hline Four- & Mean & 1.0 & 1.7 & 2.7 & 3.7 & 3.9 & 4.3 & 4.8 & 6.4 & 5.7 & 6.0 & 6.1 & 8.4 & 7.6 & 6.8 & 7.9 & 8.8 \\
\hline Block & SD & .1 & .4 & .6 & .6 & 1.2 & 1.5 & 1.9 & 1.9 & 2.8 & 3.3 & 3.2 & 3.5 & 3.8 & 4.4 & 4.8 & 4.6 \\
\hline Two- & Mean & 1.0 & 1.7 & 2.5 & 3.7 & 4.2 & 5.5 & 5.4 & 6.6 & 6.9 & 8.6 & 8.0 & 9.7 & 8.7 & 11.2 & 8.8 & 12.2 \\
\hline Block & $\mathrm{SD}$ & .0 & .3 & .6 & .5 & 1.0 & 1.0 & 1.6 & 2.0 & 2.4 & 2.0 & 3.4 & 2.9 & 3.9 & 3.4 & 4.8 & 4.0 \\
\hline & Mean & 1.0 & 1.9 & 2.7 & 3.7 & 4.5 & 5.4 & 5.6 & 6.8 & 7.4 & 8.1 & 8.3 & 9.6 & 9.7 & 10.0 & 10.5 & 10.8 \\
\hline Random & SD & .0 & .3 & .6 & .8 & 1.0 & 1.1 & 1.9 & 1.7 & 2.2 & 2.4 & 3.1 & 2.9 & 3.3 & 4.1 & 4.6 & 4.6 \\
\hline
\end{tabular}




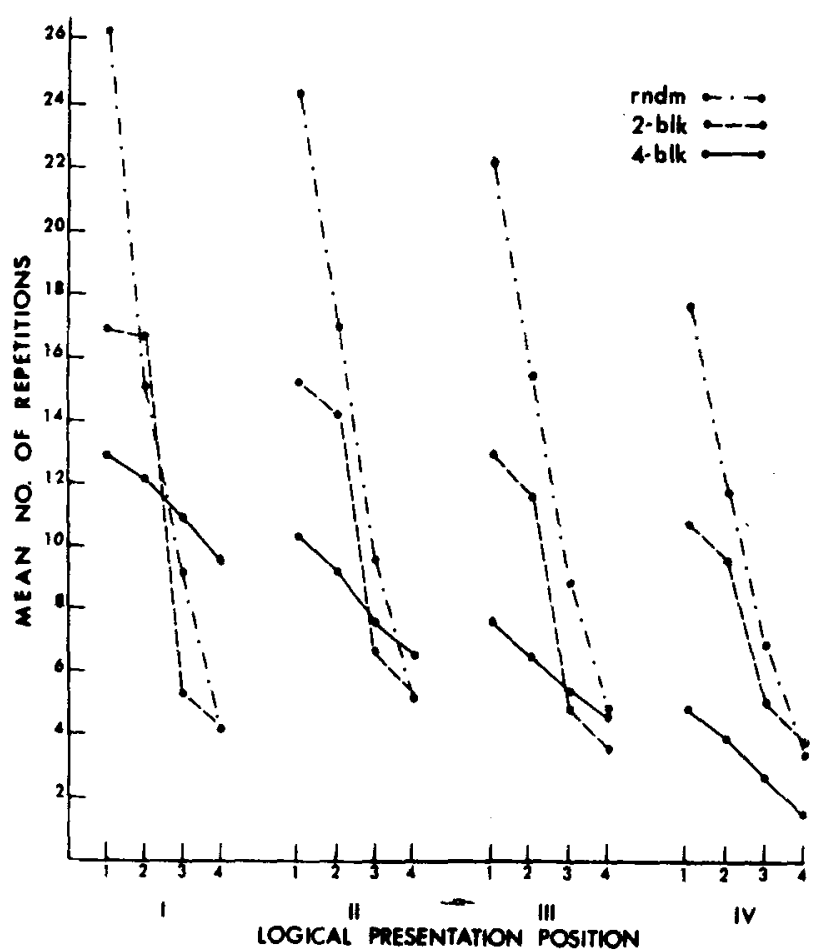

Figure 1. Overt repetitions for Sequential Organization groups across logically reordered positions within and across categories.

("horse") trigger the rehearsal of already presented items ("dog, cat, cow"), then rehearsal set sizes should increase across input positions. The analysis confirmed this effect $[F(15 / 810)=88.70, p<.001, \bar{x}=.08]$. The interaction of input position with sequential organization $[F(30 / 810)=2.63, p<.001, s \bar{x}=.08]$ further showed that this effect differed as a function of a priori list organization (see Table 1, Part D). The 4-block groups exhibited peaks at category boundaries in the number of different items rehearsed; the 2-block and random conditions also exhibited rehearsal set patterns isomorphic with their input time patterns. Clearly, overt rehearsal was not restricted to an item's initial presentation, but was distributed across the entire list.

The rehearsal hypothesis predicts that decreasing item repetitions within and across categories should be obtained during input, due to the cueing function of later presented category exemplars. The same rehearsal patterns should also be observed when order and speed of output are considered, thus providing evidence for a correspondence between repetition and retrieval processes. Note, however, that any occurrence of these retrieval patterns would be obscured in the above results, since the analyses examined successive input positions without regard to category membership. In other words, patterns of input across objective input positions for 2-block and random conditions bear no direct relationship to the rehearsal patterns predicted in the introductory remarks. A more satisfactory procedure would involve reorganizing the items on the joint basis of category membership and relative order of presentation, thereby allowing any relationships between item rehearsals and the categorized structure of the lists to be revealed. A similar procedure, conditionalizing item repetitions on output order, should demonstrate the relationships between rehearsal and retrieval characteristics.

In order to examine directly the patterned rehearsal of items and categories during input, the overt repetition data for free recall groups were reordered according to "logical presentation position." This procedure blocked the four exemplars of the same category together according to their relative order of presentation, regardless of intervening noncategory members. For example, if a random list were presented in the following order-Dog, Fork, Pea, Cat, Oil, Spoon, Gas,...-the items would be logically reordered as follows: (Animals-I) Dog-1, Cat-2, -3, 4; (Kitchen Utensils-II) Fork-1, Spoon-2, -3, 4; (Vegetables-III) Pea-1, -2, -3, 4; (Fuel-IV) Oil-1, Gas-2, -3, 4. Repetitions for the 4-block condition were, of course, already in blocked order.

The results of this reordering are presented in Figure 1. The analysis revealed a significant decrease in repetitions within categories $[\mathrm{F}(3 / 81)=42.30, \mathrm{p}<.001$, $s \overline{\mathrm{X}}=.70]$. A decrease in repetitions per category across category input positions also was reliable $[F(3 / 81)=51.12, p<.001, s \bar{x}=.30]$. The significant first-order interactions of sequential organization, category input positions, and positions within categories were qualified by the triple interaction of these factors $[F(18 / 243)=5.82, p<.001, \bar{s} \bar{X}=.06]$ (see Figure 1). While all groups exhibited a decrease in repetitions across categories and across positions within categories, the interaction revealed a greater decrease in repetitions for the 2-block and random groups. In other words, early presented category exemplars were rehearsed more often as list organization decreased. Given the rehearsal set size effect in Table 1, Part D, the greater rehearsal of early presented exemplars can be localized at later input positions. As such, these results support the notion that later exemplars cue the rehearsal of already presented exemplars.

Figure 2 illustrates the relationship between repetition frequency and output order. For this figure, the number of item repetitions was conditionalized on item output position in the clustered recall sequence; reentries into previously sampled categories, comprising only $3.97 \%$ of all emitted responses, were excluded. Only the data from free recall groups were considered.

Statistical analysis confirmed that retrieval order was predictable from input repetitions. The most frequently rehearsed items within each category appeared earliest in the output of that category $[\mathrm{F}(3 / 75)=18.24, \mathrm{p}<.001$, $s \overline{\mathrm{X}}=.26]$. Further, category units with the greatest overall rehearsal were also the first categories to be recalled $[F(3 / 75)=3.58, p<.025, s \bar{x}=.34]$. In terms of retrieval order, then, these patterns of rehearsal 
strongly indicate that retrieval of both categories and items within categories proceeds from strongest to weakest. Note also that the last recalled item in a category invariably received fewer repetitions that the first item recalled in the immediately subsequent category, supporting the modification that recalling all of a category's instances takes priority over the absolute rehearsal frequency of individual items. Finally, the interaction of sequential organization with output position within categories $[\mathrm{F}(6 / 75)=4.13, \mathrm{p}<.005$, $\mathrm{s} \overline{\mathrm{X}}=.26]$ indicated that the initially recalled items of each category were rehearsed more during input when subjects were presented with less organized lists.

The correspondence between repetition patterns in Figures 1 and 2 is striking. Rehearsal and reorganization during input resulted in differential amounts of repetition, with "logically" earlier categories and items being rehearsed to a greater extent. These patterns of repetitions were duplicated in Figure 2, suggesting that the number of item repetitions directly influenced order of output within a category. A similar relationship between decreasing repetitions per category and category output order was also obtained. Clearly, then, the predictions of retrieval order based on rehearsal frequency and reorganization were confirmed. It should be emphasized again that these patterns, in combination with the cluster size results, do not support a strict serial recall, or input position, interpretation of retrieval order. Rather, the retrieval order effects seem best interpreted as jointly dependent on rehearsal frequency and reorganization during storage.

An examination of within-category IRTs according to output position revealed the predicted correspondence between repetitions and retrieval time. Figure 3 displays the average within-category IRTs plotted according to

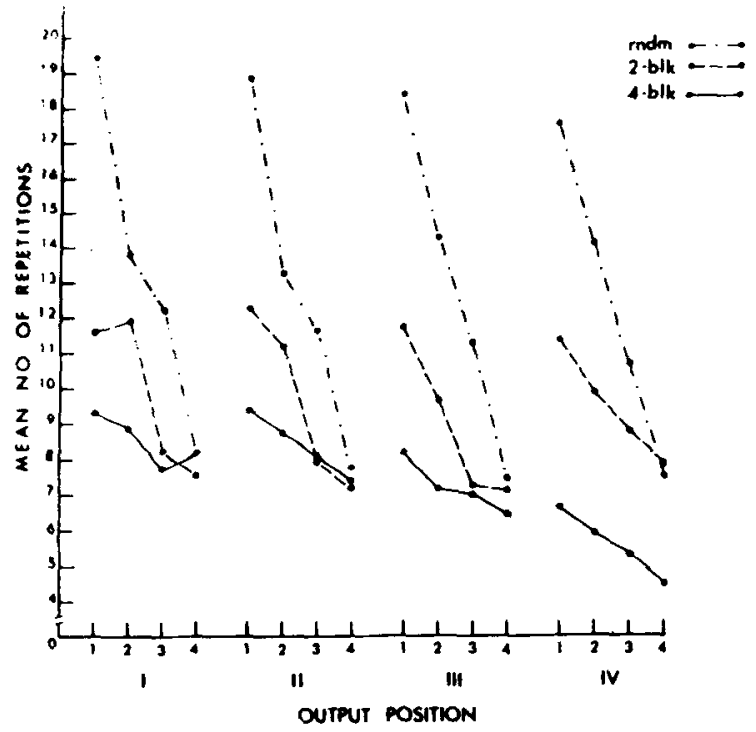

Figure 2. Overt repetitions for Sequential Organization groups conditional on output order of categories and items within categories.

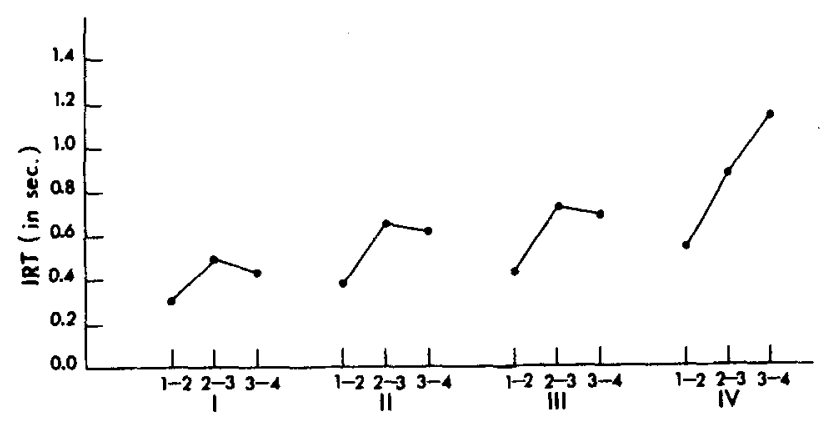

Positions within categories for the first 4 categories

Figure 3. IRTs across successive positions within categories.

output order; as in Figure 2, category reentries were excluded from consideration. Overall, within-category IRTs increased across the three positions within categories, $\quad[F(2 / 84)=6.88, p<.005, \quad \bar{s} \bar{x}=.07]$. A comparison of this increase to the decrease in repetitions within categories (Figure 2) supports the prediction that category exemplars receiving more repetitions will be retrieved more rapidly within the category. The increase in average IRTs across the four category output positions $[F(3 / 126)=11.47, \quad p<.001, \quad \bar{x}=.05]$ complements the decrease in average category rehearsal, confirming that higher order units submitted to greater rehearsal will also be retrieved more rapidly.

The effects of the rehearsal requirement on retrieval time fully supported the above relationship between repetition and retrieval. Further, these results provided additional information regarding the influence of increased serial processing on the retrieval of categorized material. Overt (vs. covert) rehearsal led to reliably longer study times during input. The effects of this increased storage processing were revealed in shorter within-category retrieval times $[F(1 / 42)=4.64, p<.05$, $\overline{\mathrm{s}}=.16]$ and a smaller increase in within-category IRTs across category output positions $[F(3 / 126)=2.84$, $\mathrm{p}<.05, \mathrm{~s} \overline{\mathrm{X}}=.05$ ] for overt groups. A similar effect was found in the comparison of between- and within-category IRTs $[F(1 / 42)=9.81, p<.005$, $s \overline{\mathbf{X}}=.35]$; that is, IRTs from overtly rehearsing groups were reliably shorter than those from covert rehearsal groups. The interaction of Rehearsal Requirement by Type of IRT by Output Position $[F(2 / 84)=5.32$, $\mathrm{p}<.01, s \overline{\mathbf{X}}=.10$ ], was also significant. Means and standard deviations for this interaction are displayed in Table 2. The interaction revealed shorter within-category IRTs across positions for overt groups, as noted above, as well as a smaller increase across positions in between-category IRTs for these groups.

According to Patterson et al. (1971), increases in the between-category IRT function reflect the time required to gain access to successive higher order units during recall. In view of the increased serial processing engendered by overt rehearsal, it is possible that overtly 
Table 2

Means and Standard Deviations (in Seconds) of Between- and Within-Category IRTs Across Three Category Output Positions, Separately for Overt and Covert Rehearsal Groups

\begin{tabular}{|c|c|c|c|c|c|c|c|}
\hline \multirow{3}{*}{$\begin{array}{l}\text { Type of } \\
\text { IRT* }\end{array}$} & & \multicolumn{6}{|c|}{ Category Output Position } \\
\hline & & \multicolumn{2}{|c|}{1} & \multicolumn{2}{|c|}{2} & \multicolumn{2}{|c|}{3} \\
\hline & & B & W & B & W & B & W \\
\hline Overt & Mean & 1.48 & .43 & 1.91 & .57 & 2.41 & .62 \\
\hline Rehearsal & $\mathrm{SD}$ & .70 & .26 & 1.31 & .34 & 1.52 & .38 \\
\hline Covert & Mean & 1.79 & .62 & 2.85 & .62 & 5.96 & .91 \\
\hline Rehearsal & $\mathrm{SD}$ & 2.20 & .36 & 2.11 & .31 & 5.84 & .53 \\
\hline
\end{tabular}

${ }^{*} B=$ between-category $I R T, W=$ within-category $I R T$.

rehearsing subjects generated a retrieval strategy which included some serial component for higher order unit retrieval. To the extent that serial information linking the categories was stored along with item information, such a strategy might lead to more rapid access to successive higher order units, and hence a smaller increase in the IRT function. This explanation has been offered elsewhere (McCauley \& Kellas, 1974), and is entirely consistent with Patterson et al.'s (1971) views concerning components of between-category IRTs. Further support for this interpretation comes from a comparison of IRTs for 4-block groups. In this analysis, the between-category IRT function for serial recall groups increased less than the comparable function for free recall groups. Again, establishing some sort of sequential associations among categories, necessary for successful serial recall of 4-block lists, seems to have decreased category access time during rehearsal.

\section{GENERAL DISCUSSION}

The results of this study support Kellas et al.'s (1973) hypothesis that rehearsal frequency exerts a direct influence over order and speed of retrieval, both within and across categories. Logical reordering of rehearsal repetitions demonstrated that early items were rehearsed to a greater extent than later items from the same category. The same pattern of rehearsal was also obtained when repetitions were conditionalized on output order, indicating a direct relationship between rehearsal frequency and retrieval order. In combination with the increasing IRT patterns across output positions, these results confirm the prediction that retrieval proceeds from most to least rehearsed, both for items within categories and for categories themselves, with more frequently rehearsed items and categories being retrieved more rapidly. It also appears that recalling all the accessible items of a category has priority over the absolute level of repetition of individual list items (Rundus, 1971). These findings, then, are entirely consistent with the proposal of similar retrieval mechanisms for higher- and lower-order memory units (Wood, 1972) as well as the general hypothesis of rehearsal frequency and reorganization as a predictor of retrieval order and speed. There is the possibility that reorganization or logical presentation position per se might determine output order, independent of rehearsal frequency. While the effects of these two factors cannot be evaluated separately here, it does seem unlikely that logical presentation position alone could also account for the present retrieval time effects.

As noted earlier, demonstration of this relationship between rehearsal frequency and retrieval order and speed is difficult to explain within a random sampling model of retrieval. As such, these results call into question those retrieval models based solely on random sampling and replacement of items and categories (e.g., Patterson, et al., 1971; Pollio, Richards, \& Lucas, 1969). Neither the between- or within-category IRT patterns suggested the operation of a recognition check component, as would be predicted by replacement models. Further, the pattern of repetitions conditionalized on output order is not amenable to any random sampling interpretation. In appears, therefore, that an adequate model of retrieval must be stated not only in terms of retrieval processes per se, but also in terms of those rehearsal and reorganization processes which affect subsequent retrieval. Given sufficient processing time, or repeated exposures of the stimuli, subjects can actively reorganize the lists, basing their retrieval on repetition frequency and semantic relationships. However, insufficient processing time, longer lists, or perhaps different levels of organization or lag between category members (see Mandler, 1973) might be expected to impede reorganization, and result in the inclusion of a recognition check component in the retrieval strategy. In both cases, however, it appears that retrieval effects can be attributed to observable storage processes and their influence on retrieval processes.

A final relevant issue concerns the nature of overt repetitions in memory tasks. Recent investigations by Craik and Watkins (1973) and Woodward, Bjork and Jongeward (1973) have demonstrated that overt repetition of list items may serve only a "maintenance rehearsal" function, that is, merely preserving an item from decay without increasing its probability of recall. "Elaborative rehearsal," on the other hand, involves a deeper semantic level of analysis, with such processes increasing subsequent retrieval performance. The present results clearly implicate semantic processing, in that list items were reorganized and recalled on the basis of membership in conceptual categories. The problem, however, concerns the nature and function of the overt repetitions observed here, and their predictive relationship to retrieval order and speed. If we assume, along with Craik and Watkins (1973), that overt repetitions may represent only "maintenance rehearsal" at a given level of analysis, then a repetition-retrieval relationship should not have been obtained. Given the strength of this relationship in the present study, 
however, we must conclude that overt repetitions were at least highly correlated with elaborative, semantic rehearsal. In effect, this conclusion affirms that overt rehearsals can serve as a reliable index of elaborative rehearsal processes.

\section{REFERENCES}

Battig, W. F., \& Montague, W. E. Category norms for verbal items in 56 categories: A replication and extension of the Connecticut category norms. Joumal of Experimental Psychology Monograph, 1969, 80, No. 3, Part 2.

Craik, F. I. M., \& Watkins, M. J. The role of rehearsal in short-term memory. Joumal of Verbal Learning and Verbal Behavior, 1973, 12, 599-607.

Fischler, I., Rundus, D., \& Atkinson, R. C. Effects of overt rehearsal processes on free recall. Psy chonomic Science, 1970, $19,249-250$

Kellas, G Ashcraft, M. H., Johnson, N. S., \& Needham, S, Temporal aspects of storage and retrieval in free recall of categorized lists. Joumal of Verbal Learning and Verbal Behavior, 1973, 12, 499-511.

Kellas, G., McCauley, C.. \& McFarland, C.E. A reexamination of externalized rehearsal. Joumal of Experimental Psychology: Human Leaming and Memory, 1975, 104, 84-90.
Mandler, G. Memory storage and retrieval: Some limits on the reach of attention and consciousness. (Tech. Rep. No, 34) San Diego: University of California, Center for Human Information Processing, 1973.

McCauley, C., \& Kellas, G. Induced chunking: Temporal aspects of storage and etrieval. Journal of Experimental Psy chology, $1974,102,260-265$.

Patterson, K. E. Meltzer, R. H \& Mandler, G. Inter-response times in categorized free recall. Joumal of Verbal Leaming and Verbal Behavior, 1971, 10, 417-426.

Pollio, H. R., Richards, S., \& Lucas, R. Temporal properties of category recall. Joumal of Verbal Learning and Verbal Behavior, 1969, 8, 529-536.

Rundus, D. Analysis of rehearsal processes in free recall. Journal of Experimental Psy chology, 1971, 89, 63-77.

Rundus, $D$. Negative effects of using list items as recall cues. Journal of Verbal Learning and Verbal Behavior, 1973, 12 , 43-50.

Wood, G. Organization processes and free recall. In E. Tulving and $W$. Donaldson (Eds.) Organization of memory. New York: Academic Press, 1972.

Woodward, A. E., Bjork, R. A., \& Jongeward, R. H. Recall and recognition as a function of primary rehearsal. Joumal of Verbal Learning and Verbal Behavior, 1973, 12, 608-617.

(Received for publication October 9.1974: revision received December 19,1974 .) 